\title{
Mejoramiento de la calidad en la recepción de materias primas, mediante la implementación de un plan de muestreo por variables
}

\section{Quality improvement in the reception of raw materials, through the implementation of a sampling plan by variables}

\author{
HERNÁNDEZ-BENITO, Fernando†, GONZÁLEZ-SÓBAL， Martín*， GÓMEZ-MÁRQUEZ, \\ Montserrat y SOLÍS-JIMÉNEZ, Miguel Ángel \\ Colegio Diocesano José Antonio González Peña, México. \\ Tecnológico Nacional de México, Instituto Tecnológico Superior de Huatusco, Maestría en Ingeniería, División Ingeniería \\ Industrial, México.
}

ID $1^{\text {er }}$ Autor: Fernando, Hernández-Benito / ORC ID: 0000-0002-3944-6849, PubMed Author ID: 240961198, Researcher ID Thomson: ABC-2759-2020.

ID $1^{\text {er }}$ Coautor: Martín, González-Sóbal / ORC ID: 0000-0003-0038-8319, Researcher ID Thomson: N-6243-2018, CVU CONACYT ID: 463431 ID $2^{\text {do }}$ Coautor: Montserrat, Gómez-Márquez / ORC ID: 0000-0002-2901-1745, Researcher ID Thomson: S-8535-2018,
CVU CONACYT: 999026

ID $3^{\text {er }}$ Coautor: Miguel Ángel, Solís-Jiménez / ORC ID: 0000-0002-8125-0989, Researcher ID Thomson: N-6243-2018, CVU CONACYT ID: 94216

DOI: $10.35429 / J I E .2020 .13 .4 .19 .31$

Recibido Julio 25, 2020; Aceptado Diciembre 30, 2020

\section{Resumen}

Objetivo: Garantizar la adecuada inspección de materia prima y material de empaque por el área de inspecciónrecibo mediante la implementación de un plan de muestreo efectivo que permita disminuir el porcentaje de materia prima defectuosa y su impacto en el proceso productivo. Metodología: Se basa en el ciclo de mejora continua (PHVA) dentro de la cual se realiza un diagnóstico para determinar el estado actual del proceso de inspección, lo que permitirá definir nuevas estrategias de acción encaminadas a estandarizar el proceso de inspección, mediante la implementación de un plan de muestreo por variables en base a la norma MIL-STD 414, una vez implementado se procede a documentar dicho proceso, a la par se realiza la integración de proveedores, mediante evaluaciones periódicas con el fin de que conozcan la dinámica del nuevo proceso de inspección y trabajar sólo con aquellos proveedores comprometidos con la calidad de sus insumos. Contribución: Reducción del porcentaje de materia prima defectuosa, de un $21 \%$ a un $13 \%$, lo que significa un nivel de calidad aceptable de los materiales de un $87 \%$.

Plan de muestreo, Nivel de calidad aceptable, Proveedores

\begin{abstract}
Objective: To guarantee the adequate inspection of raw material and packaging material by the inspection-receipt area by implementing an effective sampling plan that allows reducing the percentage of defective raw material and its impact on the production process. Methodology: It is based on the continuous improvement cycle (PHVA) within which a diagnosis is made to determine the current state of the inspection process, which will allow defining new action strategies aimed at standardizing the inspection process, through the implementation of a sampling plan by variables based on the MIL-STD 414 standard, once implemented, this process is documented, at the same time the integration of suppliers is carried out, through periodic evaluations in order to know the dynamics of the new inspection process and work only with those suppliers committed to the quality of their inputs. Contribution: Reduction of the percentage of defective raw material, from $21 \%$ to $13 \%$, which means an acceptable quality level of the materials of $87 \%$.
\end{abstract}

Sampling plan, Acceptable quality level, Supplier

Citación: HERNÁNDEZ-BENITO, Fernando, GONZÁLEZ-SÓBAL, Martín, GÓMEZ-MÁRQUEZ, Montserrat y SOLÍSJIMÉNEZ, Miguel Ángel. Mejoramiento de la calidad en la recepción de materias primas, mediante la implementación de un plan de muestreo por variables. Revista de Ingeniería Industrial. 2020. 4-13:19-31.

\footnotetext{
*Correspondencia al Autor (Correo Electrónico: martinglezs@itshuatusco.edu.mx)

$\dagger$ Investigador contribuyendo como primer autor.
} 


\section{Introducción}

Esta investigación se enfoca en implementar un plan de muestreo de aceptación por variables, identificando las materias primas y materiales de empaque adecuados para la fabricación de productos dentro de una empresa, que se dedica a la manufactura textil de contenedores de polipropileno, en el municipio de Paraje Nuevo, Córdoba, Veracruz, México. Este proyecto se ejecutó durante el segundo semestre del año 2019.

En la actualidad la empresa se rige bajo un sistema de calidad en donde al recibir materiales para la producción, se inspecciona solo un $10 \%$ de ellos, obteniendo resultados poco favorables, puesto que la calidad no es adecuada y en ocasiones estos materiales, se desechan o reutilizan para actividades extras, ocasionando retrasos en la producción, y producto terminado de baja calidad.

De lo anterior surge la necesidad de implementar una herramienta de mejora para el proceso de recepción de materiales, basada en el ciclo de mejora continua PHVA, dicha técnica corresponde a diseñar un plan de muestreo para aceptación por variables, su aplicación permitirá a la empresa recibir lotes de materia prima en grandes cantidades.

Para lograr lo anterior, en primer lugar, se evalúa el método actual de inspección, con el fin de conocer su nivel de eficiencia, así mismo se realiza una clasificación de proveedores, partiendo del nivel de calidad de sus insumos. En segundo lugar, se diseña e implementa el plan de muestreo por variables, tomando como base la norma MIL-STD-414, lo que permitirá definir el tamaño de muestra a inspeccionar en diferentes tamaños de lotes, así mismo definir criterios de aceptación o rechazo de los mismos.

Se evaluaron 27 embarques con el plan de muestreo propuesto, lo cual trajo grandes beneficios para la empresa, en comparación con el método de inspección anterior. En cuanto al nivel de calidad aceptable (NCA) de los insumos, este mejoró de un $79 \%$ a un $87 \%$ comparando el primer y segundo semestre del año 2019, respectivamente.
En relación al desempeño de los proveedores, su calificación global comparando los años 2018 y 2019, paso de $89.375 \%$ a $85.92 \%$ respectivamente, esta disminución se debe a que antes de la implementación del plan de muestreo, no se realizaba una evaluación tan exhaustiva como la actual, sin embargo, los proveedores mantienen el compromiso de mejorar la calidad de sus insumos.

\section{Revisión de literatura}

De acuerdo con (Barra, 2012), la calidad es dar al cliente o a la siguiente persona en el proceso lo que requiere ya sea un bien o un servicio adecuado para su uso y hacer esto de tal manera que cada tarea se realice correctamente desde la primera vez y a un adecuado equilibrio económico.

La obligación principal del productor y del vendedor es asegurar al comprador el desempeño y economía de los productos y servicios de una manera efectiva, tal como lo menciona Armand V. Feigenbaum (1991), y cuando éste no se ha llevado a cabo, es la obligación del productor y vendedor, no del comprador, el arreglar las cosas y cargar con el costo.

Según Salinas (2014), una muestra está constituida por sujetos o unidades de observación, a partir de los cuales se hará inferencia al resto de la población no estudiada; es muy importante la selección adecuada de la muestra para lograr representatividad de la población de interés, de lo contrario los hallazgos de la investigación estarían sesgados $\mathrm{u}$ orientados a ciertas características.

\section{Muestreo de aceptación por variables}

En los planes de muestreo de aceptación por variables se especifican el número de artículos que hay que muestrear y el criterio para juzgar los lotes cuando se obtienen datos de las mediciones respecto a la característica de calidad que interesa, ya que como comenta Juan C. Sandoval (2010), estos planes se basan generalmente en la media y desviación estándar maestrales de la característica de calidad. 
Cuando se conoce la distribución de la característica en el lote o el proceso, es posible diseñar planes de muestreo por variables que tengan riesgos especificados de aceptar y de rechazar lotes de una calidad dada

\section{Muestreo de aceptación según MIL-STD 414}

Las tablas MIL - STD 414 proporcionan el tamaño de la muestra y el valor crítico de aceptación, para el muestreo de aceptación de productos con especificaciones superior, inferior o ambas, tanto para procesos con variabilidad conocida como desconocida, tal como lo menciona Rendón Castaño (2013).

Así mismo, utilizan el método de la desviación y el método del rango o recorrido, bajo dos modalidades, denominadas: forma $\mathrm{M} \mathrm{y}$ forma K. Adicionalmente, contemplan el diseño de planes para cinco niveles de inspección.

En este tipo de planes se toma una muestra aleatoria del lote y a cada unidad de la muestra se le mide una característica de calidad de tipo continuo (longitud, peso, espesor, etc.), y en contraste con el muestreo por atributos, no se trata de clasificar a cada unidad como defectuosa o no, sino de registrar la medición de cada pieza. Con base en estas mediciones, se calcula un índice (estadístico) que de acuerdo con su valor se aceptará o rechazará todo el lote. Por lo general, el índice toma en cuenta la información muestral (media, desviación estándar) y las especificaciones de la característica de calidad.
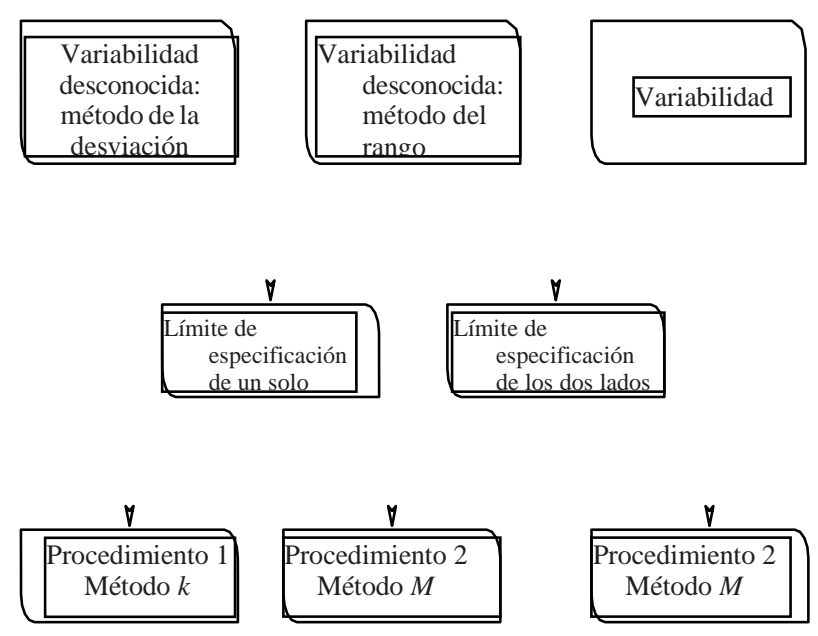

Figura 1 Organización del MIL STD 414

Fuente: (Gutiérrez Pulido \& de la Vara Salazar, Control estadístico de la Calidad y Seis Sigma, 2013).
Con base a Humberto \& Román (2013), el MIL STD 414 es un plan para muestreo de aceptación por variables lote por lote. El punto principal de este estándar es el nivel de calidad aceptable (NCA o AQL), y comprende porcentajes que van de 0.04 a $15 \%$. El estándar tiene cinco niveles generales de inspección; al nivel IV se le considera el "usual".

La MIL-STD-414 está estructurada de una forma parecida a la MIL-STD-105E. Utiliza el concepto de NCA, cuenta con cinco niveles de inspección (I - V) y niveles de inspección normal, reducida y rigurosa. Si no se indica otra cosa el nivel a aplicar es el IV. Consta a su vez de cuatro secciones A, B, C y D.

\section{Metodología para crear un plan MIL-STD 414}

1. Determinar el tamaño del lote.

2. Especificar el NCA (o AQL).

3. Escoger el nivel de inspección, usualmente el nivel IV, que puede ser cambiado si la situación lo justifica.

4. En la tabla 1 y de acuerdo con el tamaño de lote y el nivel de inspección, encontrar la letra código del tamaño de la muestra.

5. Posteriormente en la tabla 2 y de acuerdo con la letra código y el NCA, se busca el plan simple para inspección normal, que consiste en un tamaño de muestra $\mathrm{n}$, y del valor $\mathrm{M}$, que es el porcentaje máximo de defectuosos tolerado en el lote.

6. En la misma tabla 2 y partiendo de los NCA que están en la parte inferior, se encuentra el plan que se emplearía bajo inspección severa, con sus correspondientes valores para n y $\mathrm{M}$.

7. Seleccionar de manera aleatoria una muestra de tamaño $n$, y a cada pieza de ésta se le mide la característica de calidad. Con los datos obtenidos calcular la media (X) y la desviación estándar muestral (S).

8. A partir de las fórmulas siguientes, de acuerdo con el tipo de especificaciones que tenga la característica de calidad, calcular los índices ZES y ZEI. 


$$
\begin{aligned}
& \mathrm{ZES}=(\mathrm{ES}-\bar{X}) / \mathrm{S} \\
& \mathrm{ZEI}=(\bar{X}-\mathrm{EI}) / \mathrm{S}
\end{aligned}
$$

9. Estimar la proporción de unidades defectuosas en el lote. Para ello, en la tabla 3, en la columna de ZEI y ZES, se debe ubicar su correspondiente valor y desplazarse por ese renglón hasta la columna del tamaño de muestra del plan de inspección, n. El valor que se localice en la intersección valor de $\mathrm{Z}$ y $\mathrm{n}$, corresponde a la estimación del porcentaje de defectuosos del lote de lado inferior, pi, o del lado superior, $p s$, respectivamente.

10. Decisión de aceptación o rechazo (Gutiérrez Pulido \& de la Vara Salazar, Control estadístico, 2013):

Para variables sólo con especificación inferior. Aceptar el lote si pi es menor o igual al valor de M. En caso contrario rechazarlo.

- Para variables sólo con especificación superior. Aceptar el lote si ps es menor o igual que M. En caso contrario rechazarlo.

Para variables con doble especificación. Aceptar el lote si la suma de los porcentajes inferior y superior, $p=p i+p s$, es menor o igual que M. En caso contrario rechazar el lote.

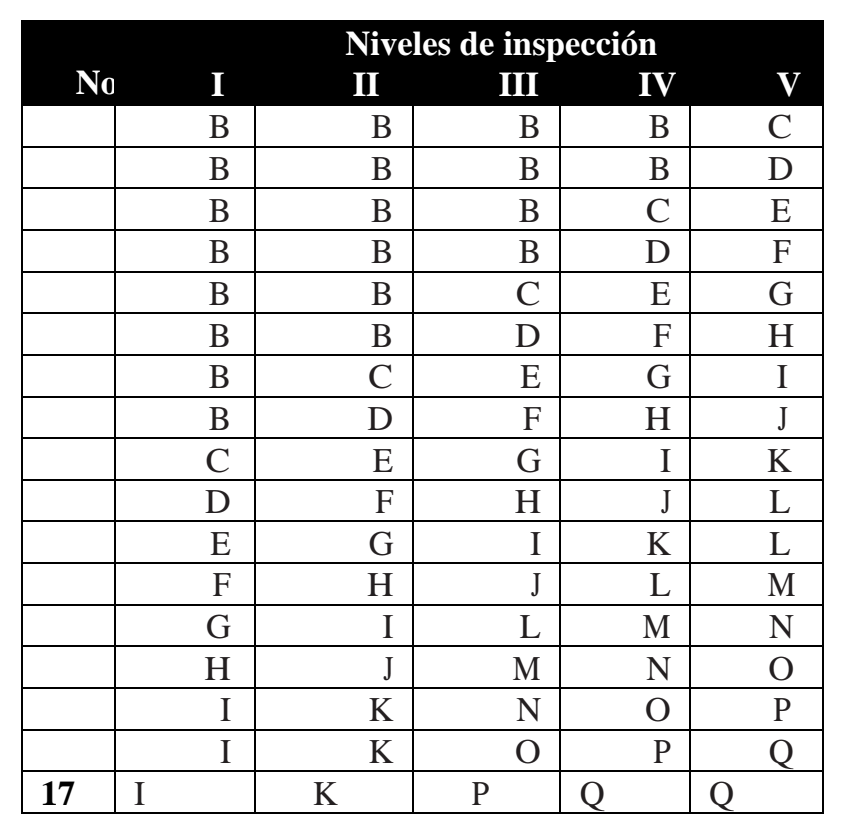

Tabla 1 Nivel de Inspección de la MIL STD 414 Fuente: (Gutiérrez Pulido \& de la Vara Salazar, Control

\begin{tabular}{|c|c|}
\hline Codigo & Muestra \\
\hline B & 3 \\
\hline $\bar{C}$ & 4 \\
\hline $\bar{D}$ & 5 \\
\hline $\mathbf{E}$ & 7 \\
\hline $\mathbf{F}$ & 10 \\
\hline G & 15 \\
\hline H & 20 \\
\hline I & 25 \\
\hline $\mathbf{J}$ & 30 \\
\hline $\mathbf{K}$ & 35 \\
\hline $\mathbf{L}$ & 40 \\
\hline $\mathbf{M}$ & 50 \\
\hline $\mathbf{N}$ & 75 \\
\hline $\mathbf{O}$ & 100 \\
\hline $\mathbf{P}$ & 150 \\
\hline $\mathbf{Q}$ & 200 \\
\hline
\end{tabular}
estadístico de la Calidad y Seis Sigma, 2013)
Tabla 2 Letras Códigos para el tamaño de muestra de la MIL STD 414

Fuente: (Gutiérrez Pulido \& de la Vara Salazar, Control estadístico de la Calidad y Seis Sigma, 2013)

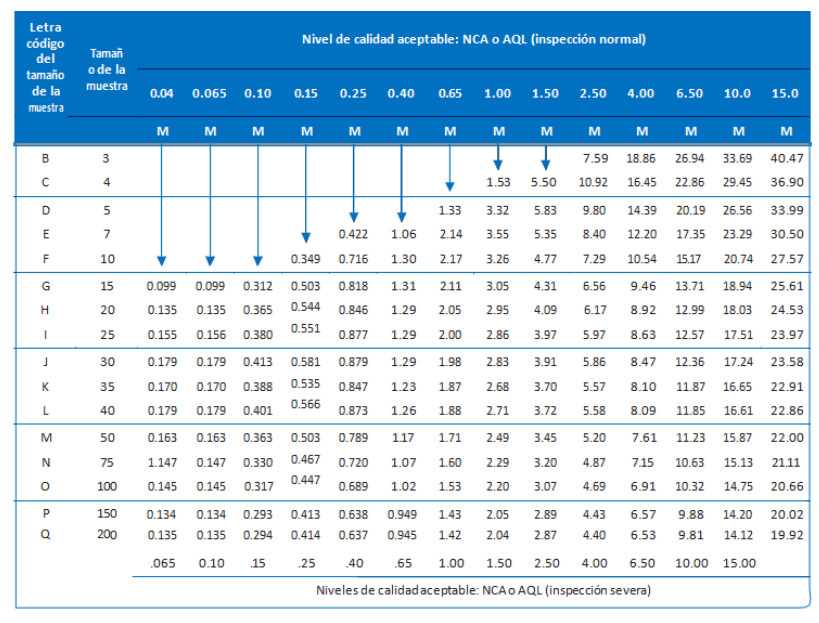

Tabla 3 Tabla para inspección normal y severa (variabilidad desconocida, método de la desviación estándar), método M.

Fuente: (Gutiérrez Pulido \& de la Vara Salazar, Control estadístico de la Calidad y Seis Sigma, 2013)

\section{Metodología}

Esta investigación es de tipo mixta, lo que permitirá evaluar el proceso de inspección de materias primas, sobre una metodología cuantitativa, mientras que el enfoque cualitativo nos presentará el nivel de desempeño de los proveedores, en relación con la calidad de sus insumos. Todo esto bajo la guía del enfoque de mejora continua, que a continuación se presenta. 


\section{Fase: Planear}

El siguiente esquema presenta la forma actual en que se realiza el proceso de inspección y análisis de materia prima:

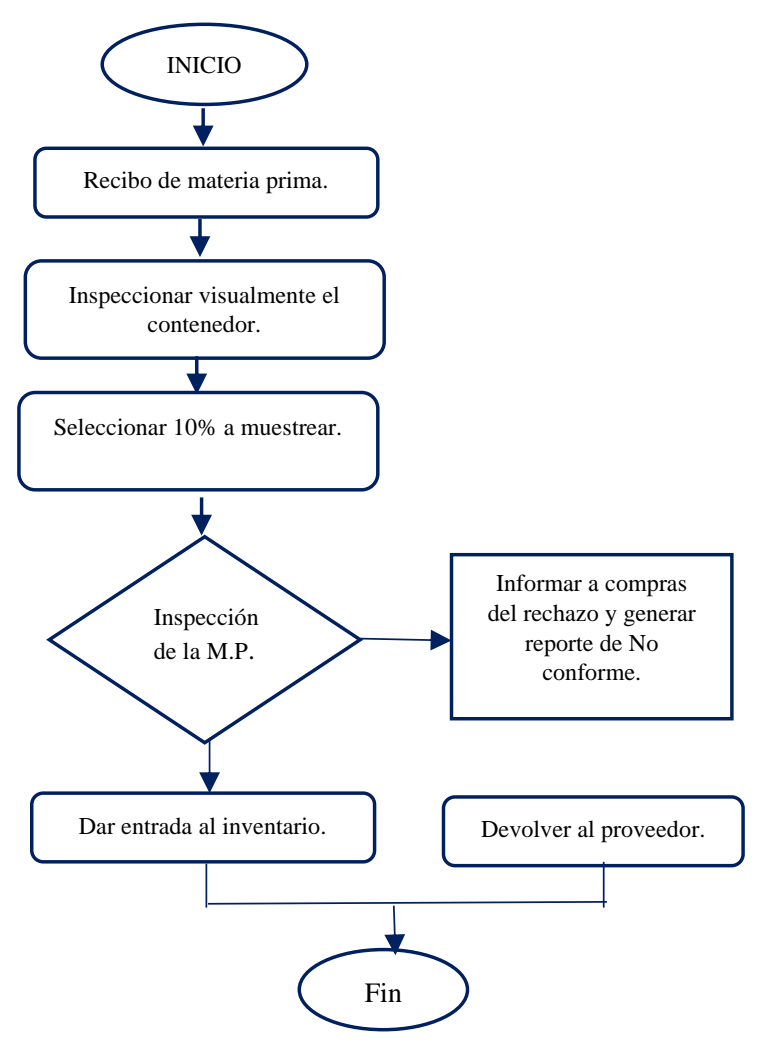

Figura 2. Método de la Inspección al 10\% Fuente: Elaboración propia.

Del proceso anterior, se evalúa su desempeño durante el primer semestre del año 2019, a continuación, se presentan los resultados obtenidos, por cuestiones de confidencialidad, se presentan sólo algunos de los insumos empleados por la empresa.

Inspección de tela.

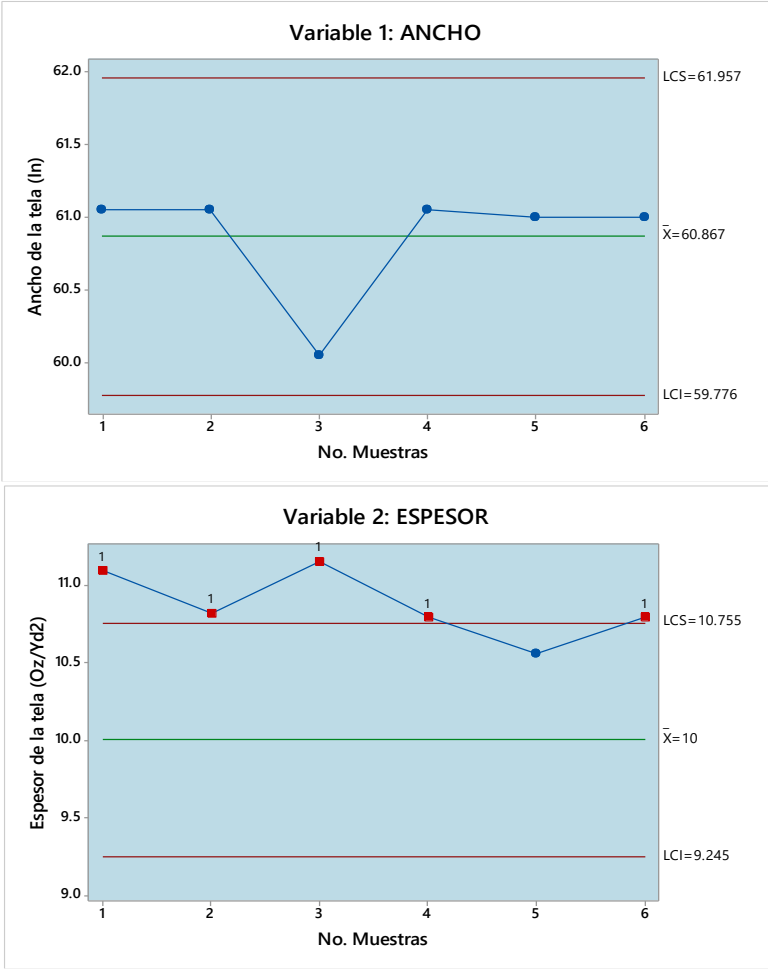

Figura 3 Inspección de tela semestre 1, 2019 Fuente: Elaboración propia

Los gráficos anteriores se realizaron en el programa MINITAB, podemos darnos cuenta, que la decisión de aceptar o rechazar dicho lote, resulta un tanto compleja, debido a que el material respecto a la variable "ancho" tuvo un registro de 60.8" y fue aprobado, al encontrar que las muestras tomadas se encontraban dentro de LE, es decir, entre 59.7" y 61.9". Analizando la variable "espesor", considerada la más crítica en este insumo, obtuvo un registro de 10 $\mathrm{Oz} / \mathrm{Yd}^{2}$, las muestras posteriores tuvieron un desempeño por encima de los LE $\left(9.2 \mathrm{Oz} / \mathrm{Yd}^{2}\right.$ a $\left.10.7 \mathrm{Oz} / \mathrm{Yd}^{2}\right)$, generando un defecto en la materia prima solicitada y un riesgo para la maquinaria.

\section{Inspección de liner.}



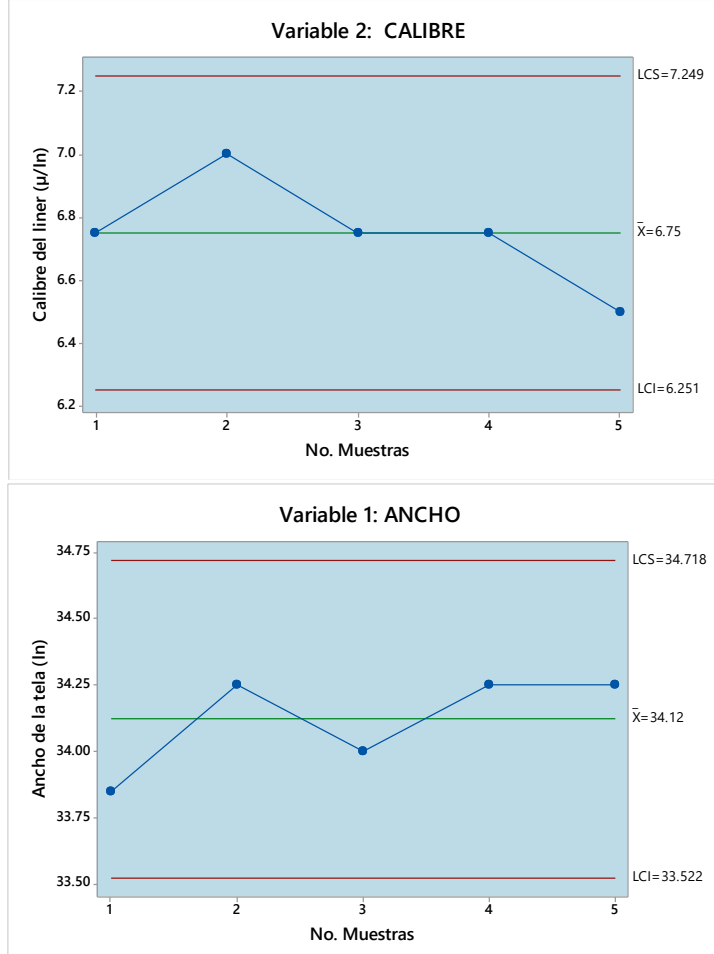

Figura 4 Inspección de liner semestre 1, 2019

Fuente: Elaboración propia

De acuerdo con la figura 4, podemos ver que la inspección del liner cumple los LE, tanto en la variable "ancho" al estar dentro de los límites permisibles (33.5" a 34.7"), como también en la variable "calibre" $(6.7 \mu / \mathrm{In})$, al estar las muestras dentro de los límites aceptables $(6.2 \mu / \mathrm{In}$ a $7.2 \mu / \mathrm{In})$.

\section{Inspección de cartón}

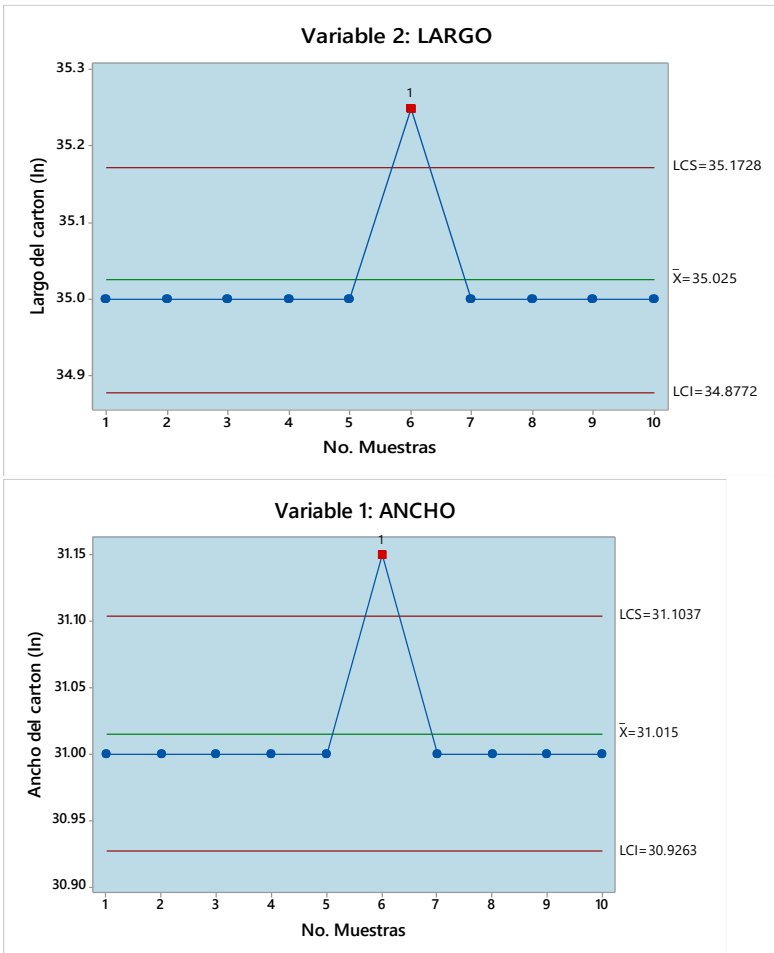

Figura 5 Inspección de Cartón 1er semestre 2019 Fuente: Elaboración propia
De acuerdo con los gráficos anteriores, podemos ver que el cartón mantenía la variable "ancho" en 31" y sus muestras estaban cerca de esa magnitud, pero una muestra superó los LE permitidos (30.9" a 31.1") por lo cual se procedió a revisar la variable "largo", la cual debería medir 35" encontrándose que una muestra superaba él LES de 35.17", por lo que el producto no cumple las especificaciones requeridas y tampoco es apto para el proceso productivo.

Durante la ejecución del diagnóstico, en el primer semestre del año 2019, se recibieron 148 lotes de materia prima y material de empaque, de los cuales 39 de ellos mostraban algún tipo de defecto por parte del proveedor, lo que representa un $21 \%$ de los embarques recibidos.

En relación con lo anterior, se realizó una encuesta para evaluar el desempeño de los proveedores que fungieron como tal, durante el último semestre del año 2018 y a la fecha. Los resultados obtenidos se presentan en el siguiente gráfico:

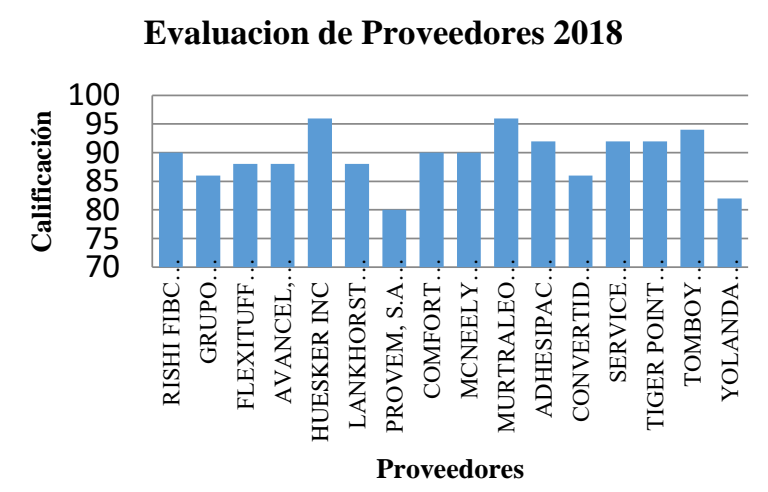

Figura 6 Grafica de Evaluación de Proveedores 2018. Fuente: Elaboración propia

Del gráfico anterior podemos observar el desempeño de los proveedores en una escala del 0 al 100, respecto al grado de cumplimiento de las especificaciones requeridas, teniendo una calificación promedio de 89.37.

\section{Etapa: Hacer}

Partiendo del diagnóstico de la situación actual, se definen las estrategias que se implementaran para mejorar el proceso de inspección de materia prima, estas se centran en dos ejes principales: definición de criterios para inspección visual y el diseño del plan de muestreo por variables en base a la norma MIL-STD 414.

HERNÁNDEZ-BENITO, Fernando, GONZÁLEZ-SÓBAL, Martín, GÓMEZ-MÁRQUEZ, Montserrat y SOLÍS-JIMÉNEZ, Miguel Ángel. Mejoramiento de la calidad en la recepción de materias primas, mediante la implementación de un plan de muestreo por variables. Revista de Ingeniería Industrial. 2020 


\section{Criterios para inspección visual.}

Se definen criterios para la verificación visual de materia prima de acuerdo con los aspectos de calidad e inocuidad, los cuales se verificarán al ingresar un nuevo contenedor al área de desembarque de la planta, entre los cuales están:

1. Inocuidad del empaque de la materia prima: Se evalúa la presentación del empaque y protección del material, si el lote llega con protección alrededor de cada caja o paca, y sobre todo que el empaque llega sin defectos, limpio $\mathrm{y}$ en condiciones inocuas.

2. Identificación de la materia prima: Si cumple con una etiqueta de identificación del material, y que esta contenga los datos adecuados como lo es: tipo de material, lote al que pertenece, dimensiones $\mathrm{o}$ especificaciones del producto, y si se encuentra visible.

3. Estibado y embalaje de la materia prima: Se verifica el acomodo o estibación del material dentro del embarque o contenedor, que cuente con tarima si es necesario, sin daños por montacargas o si se encuentra con defectos por no venir flejado el material.

4. Transporte de la materia prima: Respecto a este criterio, se incluyen los golpes, perforaciones, material dañado por transporte o por defectos en el contenedor.

Plan de muestreo.

De acuerdo con la MIL-STD-414 y su tabla de secciones se implementará la sección $\mathrm{B}$ para realizar dicho muestreo, debido a que se realizara una descripción normal de cada una de las materias primas que se reciben. A continuación, se presenta el desarrollo de dicho plan:

\section{a) Definición de la población.}

Los materiales con los que se trabaja en el área de recibo son:

\begin{tabular}{|c|c|c|c|}
\hline MATERIAL & $\begin{array}{c}\text { AREA DE } \\
\text { APLICACION }\end{array}$ & SUBCANTIDAD & $\begin{array}{l}\text { CANTIDAD } \\
\text { TOTAL/ }\end{array}$ \\
\hline Tela & $\begin{array}{l}\text { Dunnage/ } \\
\text { IBC/ Chutes / } \\
\text { Geotextil. }\end{array}$ & N/A & 120 Piezas \\
\hline Liner & $\begin{array}{l}\text { Dunnage / } \\
\text { IBC }\end{array}$ & $\mathrm{N} / \mathrm{A}$ & 30 Piezas \\
\hline $\begin{array}{l}\text { Cartón - } \\
\text { Esquineros }\end{array}$ & $\begin{array}{l}\text { Dunnage/ } \\
\text { IBC / Chutes } \\
\text { / } \\
\text { Geotextil. }\end{array}$ & 15 Estibas & $\begin{array}{l}15,000 \\
\text { Piezas }\end{array}$ \\
\hline $\begin{array}{l}\text { Cartón } \\
\text { Cajas }\end{array}$ & $\begin{array}{l}\text { Dunnage/ } \\
\text { IBC / Chutes } \\
\text { / } \\
\text { Geotextil. }\end{array}$ & 12 Estibas & $\begin{array}{l}1,000 \\
\text { Piezas }\end{array}$ \\
\hline Hilo & $\begin{array}{l}\text { Dunnage / } \\
\text { IBC / Chutes } \\
\text { / } \\
\text { Geotextil. }\end{array}$ & 6 Estibas & N/A \\
\hline Gasket & Dunnage & 100 Cajas & $\begin{array}{l}70,000 \\
\text { Piezas }\end{array}$ \\
\hline Tarimas & $\begin{array}{l}\text { Dunnage / } \\
\text { IBC / Chutes } \\
\text { / } \\
\text { Geotextil } \\
\end{array}$ & N/A & 120 Piezas \\
\hline Válvulas & Dunnage & 500 Cajas & $\begin{array}{l}450,000 \\
\text { Piezas }\end{array}$ \\
\hline $\begin{array}{l}\text { Porta- } \\
\text { documentos }\end{array}$ & N/A & 50 Cajas & $\begin{array}{l}50,000 \\
\text { Piezas }\end{array}$ \\
\hline Fleje & $\begin{array}{l}\text { Dunnage / } \\
\text { IBC / Chutes }\end{array}$ & 30 bobinas & N/A \\
\hline
\end{tabular}

Tabla 1 Materiales y Cantidades manejadas en la empresa Fuente: Elaboración propia

\section{b) Elección del tamaño de lote}

Al recibir el material el inspector del área de recibo realizará una inspección visual, verificando el tamaño de lote que está recibiendo, de acuerdo con la siguiente tabla:

\begin{tabular}{|r|r|}
\hline No. & TAMAÑO DEL LOTE \\
\hline $\mathbf{1}$ & 3 a 8 \\
\hline $\mathbf{2}$ & 9 a 15 \\
\hline $\mathbf{3}$ & 16 a 25 \\
\hline $\mathbf{4}$ & 26 a 40 \\
\hline $\mathbf{5}$ & 41 a 65 \\
\hline $\mathbf{6}$ & 66 a 110 \\
\hline $\mathbf{7}$ & 111 a 180 \\
\hline $\mathbf{8}$ & 181 a 300 \\
\hline $\mathbf{9}$ & 301 a 500 \\
\hline $\mathbf{1 0}$ & 501 a 800 \\
\hline $\mathbf{1 1}$ & 801 a 1300 \\
\hline $\mathbf{1 2}$ & 1301 a 3200 \\
\hline $\mathbf{1 3}$ & 3201 a 8000 \\
\hline $\mathbf{1 4}$ & 8001 a 22000 \\
\hline $\mathbf{1 5}$ & 22001 a 110000 \\
\hline $\mathbf{1 6}$ & 110001 a 550000 \\
\hline $\mathbf{1 7}$ & 550 001 y más \\
\hline & \\
\hline
\end{tabular}

Tabla 2 Tamaño del lote de la MIL STD 414 Fuente: (Gutiérrez Pulido \& de la Vara Salazar, Control estadístico de la Calidad y Seis Sigma, 2013).
HERNÁNDEZ-BENITO, Fernando, GONZÁLEZ-SÓBAL, Martín, GÓMEZ-MÁRQUEZ, Montserrat y SOLÍS-JIMÉNEZ, Miguel Ángel. Mejoramiento de la calidad en la recepción de materias primas, mediante la implementación de un plan de muestreo por variables. Revista de Ingeniería Industrial. 2020 


\section{c) Elegir el tipo de inspección.}

Una vez que se conoce el número de rango en el que se encuentra el lote recibido, se designa el código y nivel de inspección, recordemos que el más usado, es el nivel IV, esto de acuerdo con la tabla 1.

\section{d) Elección del tipo de inspección.}

Una vez definido el código y nivel de inspección, se procede a determinar el tamaño de muestra que se inspeccionará, esto de acuerdo con la tabla 2.

\section{e) Determinar el nivel de calidad aceptable (NCA)}

En este punto tomamos la tabla 3, se recomienda utilizar una inspección al $2.5 \%$ de porcentaje de defectos (NCA), con el código de tamaño de muestra y el NCA se determina el factor $\mathrm{M}$.

\section{f) Análisis de muestra.}

Definidos los factores del inciso anterior y con ayuda las fórmulas 1 y 2 , se determinan los límites de rechazo y aceptación para cada lote.

\section{g) Toma de decisión}

Una vez determinados los porcentajes $p i$ y $p s$, contra el valor $\mathrm{M}$, se toma el siguiente criterio de decisión:

Si $p i+p s>\mathrm{M}$, se rechaza el lote. Si $p i+p s<\mathrm{M}$, se acepta el lote.

\section{Etapa: Verificar}

\section{Aplicación de la inspección visual}

Al recibir un embarque de materia prima, se evalúa a partir del formato de inspección de calidad e inocuidad de materia prima. A partir de él se evalúa a los proveedores. Dicho formato se presenta a continuación:

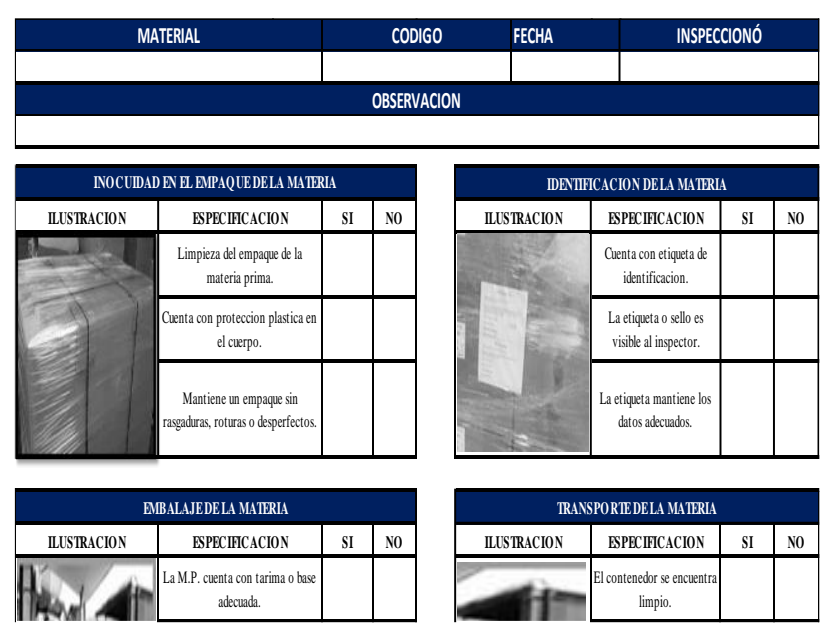

Figura 7 Criterios a Evaluar en la Inspección Visual de Inocuidad de la materia prima y AIB

Fuente: Elaboración propia

\section{Implementación del plan de muestreo}

El objetivo del plan de muestreo es determinar si las materias primas y materiales de empaque cumplen las especificaciones, por ello se implementa y verifica su funcionalidad, para finalmente estandarizar su aplicación en la planta; además de servir como herramienta metodológica al evaluar los lotes de materiales que se reciben.

A continuación, se presenta su aplicación en 3 embarques recibidos, por motivos de confidencialidad, no se puede mostrar el total de embarques evaluados.

\section{Embarque 1}

El embarque de liner que se recibe mantiene un total de 15 rollos y de acuerdo con el muestreo de aceptación a partir de la MILSTD-414, se deberá inspeccionar la cantidad de 3 rollos, debido a que se encuentra en el nivel 2, con el código de la letra $\mathrm{B}$, para finalmente los resultados compararlos con el valor de $\mathrm{M}$ que equivale a 7.59. A continuación se presenta el muestreo aplicado:

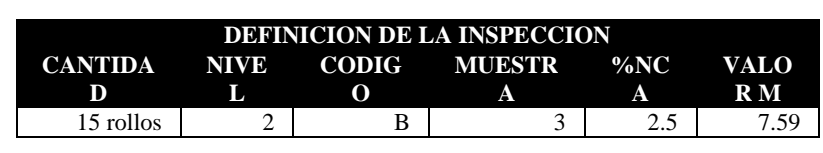

Tabla 6 Parámetros de inspección, embarque 1

Fuente: Elaboración propia. 

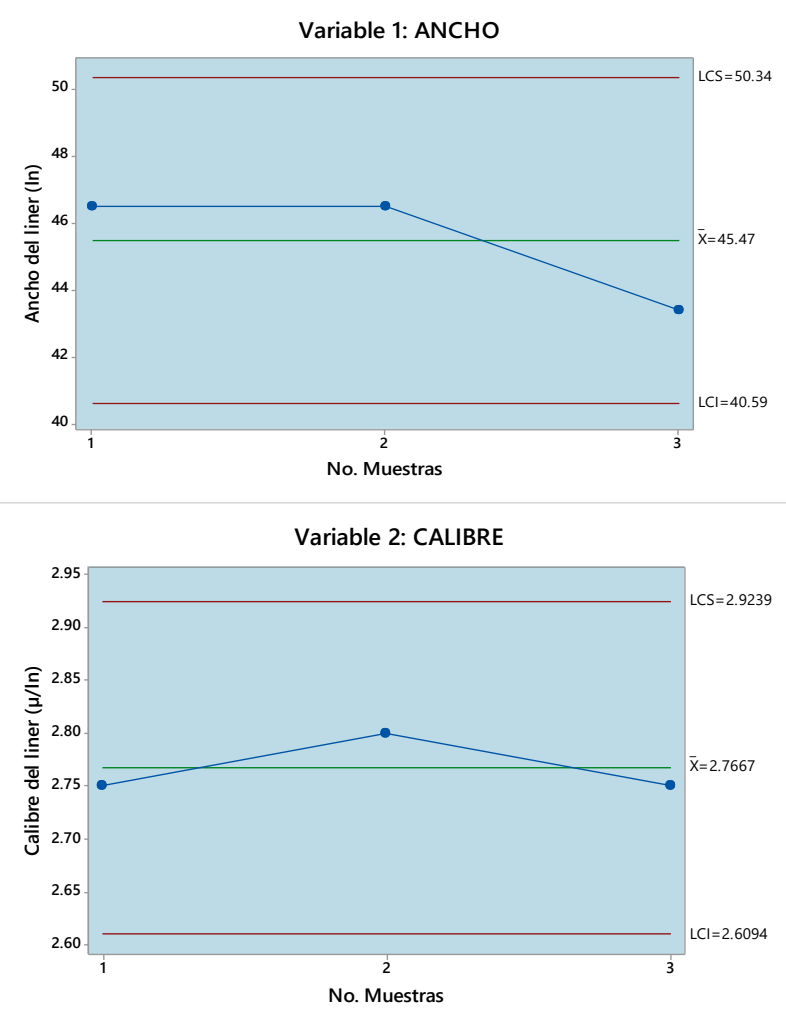

Figura 8 Gráficos de inspección, embarque 1 Fuente: Elaboración propia

\begin{tabular}{|l|r|r|l|l|r|r|}
\hline \multicolumn{7}{|c|}{ ANALISÍS DE LA INSPECCION } \\
\hline \multicolumn{1}{|c|}{ Variables } & Ancho & \multicolumn{1}{c|}{ Calibre } & \multicolumn{1}{l|}{ Variables } & Ancho & Calibre \\
\hline LSE $=$ & 50.34 & 2.923 & & ZES $=$ & 2.722 & 5.607 \\
\hline LIE $=$ & 40.59 & 2.609 & & ZEI $=$ & 2.727 & 5.607 \\
\hline$\dot{X}=$ & 45.47 & 2.766 & & PS $=$ & 0 & 0 \\
\hline $\mathrm{s}=$ & 1.789 & 0.028 & & PI $=$ & 0 & 0 \\
\hline
\end{tabular}

Tabla 7 Análisis de inspección, embarque 1 Fuente: Elaboración propia

\section{TOMA DE DECISIONES}

\begin{tabular}{|c|c|c|c|}
\hline \multicolumn{4}{|c|}{$\begin{array}{l}\text { Sí (PS + PI < M), entonces el lote se ACEPTA, de lo contrario el lote } \\
\text { se RECHAZA. }\end{array}$} \\
\hline ANCHO & & CALIBRE & \\
\hline$(0+0<7.59)$ & ACEPTADO & $\begin{array}{r}(0+0< \\
7.59)\end{array}$ & ACEPTADO \\
\hline
\end{tabular}

Tabla 8 Criterio de decisión embarque 1

Fuente: Elaboración propia

De acuerdo con el muestreo aplicado, podemos verificar que el liner cumple con los requisitos respecto a la variable "ancho" al estar dentro de los LE (40.59" a 50.34"), así como también en la variable "calibre" al verificarla con la ayuda del micrómetro y mantener los registros dentro de los límites aceptables $(2.6 \mu /$ In a 2.9 $\mu / \mathrm{In}$ ), por lo cual es apto para la producción.

\section{Embarque 2}

El embarque de tela mantiene un total de 20 rollos y de acuerdo al muestreo de aceptación, se deberá tomar una muestra de 3 rollos, con nivel 3 de inspección, código B, para finalmente los resultados compararlos con el valor de $\mathrm{M}$ que equivale a 7.59. A continuación se presenta el muestreo aplicado:

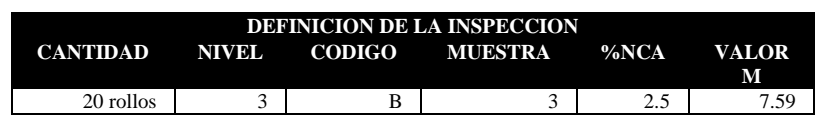

Tabla 9 Parámetros de inspección, embarque 2

Fuente: Elaboración propia

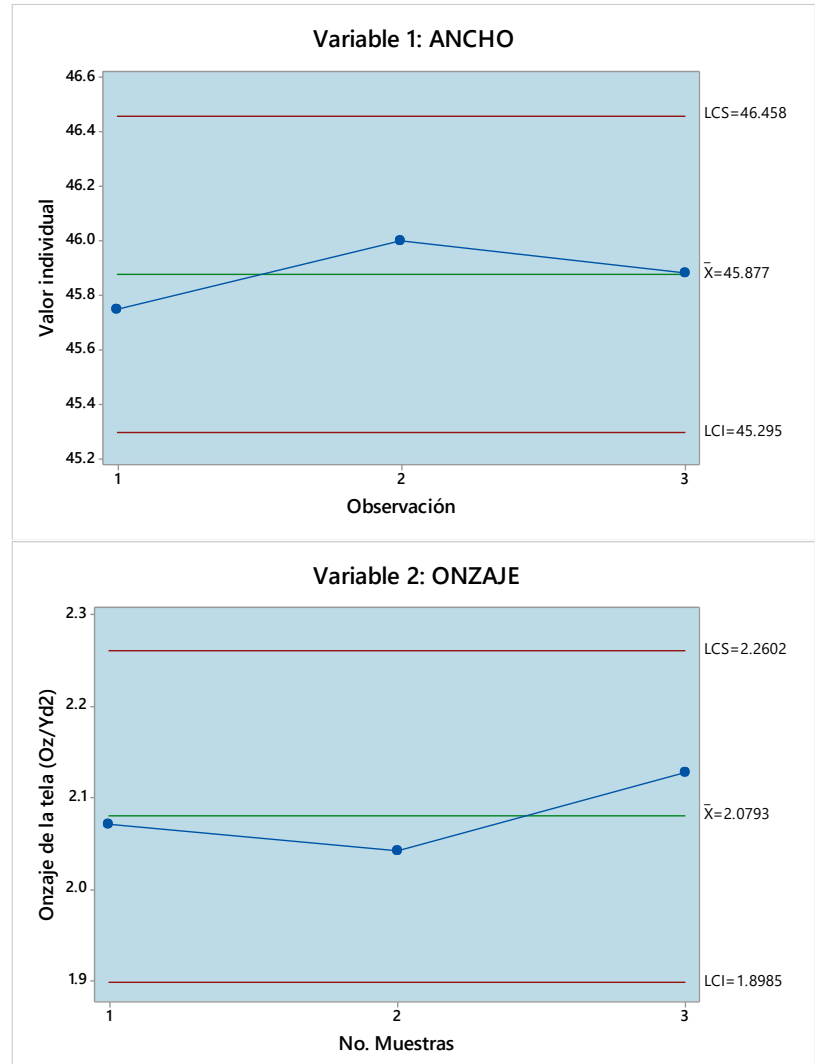

Figura 9 Gráficos de inspección, embarque 2

Fuente: Elaboración propia

\begin{tabular}{|l|r|r|l|r|r|r|}
\hline \multicolumn{7}{c|}{ ANALISÍS DE LA INSPECCION } \\
\multicolumn{1}{|c|}{ Variables } & Ancho & \multicolumn{1}{c|}{ Onzaje } & \multicolumn{1}{c|}{ Variables } & Ancho & \multicolumn{1}{c|}{ Onzaje } \\
\hline LSE $=$ & 46.458 & 2.260 & & ZES $=$ & 4.648 & 4.209 \\
\hline LIE $=$ & 45.295 & 1.898 & & ZEI $=$ & 4.656 & 4.209 \\
\hline$\dot{\mathrm{X}}=$ & 45.877 & 2.079 & & PS $=$ & 0 & 0 \\
\hline $\mathrm{s}=$ & 0.125 & 0.043 & & PI $=$ & 0 & 0 \\
\hline
\end{tabular}

Tabla 10 Análisis de inspección, embarque 2

Fuente: Elaboración propia

\begin{tabular}{|c|c|c|c|}
\hline \multicolumn{4}{|c|}{ TOMA DE DECISIONES } \\
\hline ANCHO & & ONZAJI & \\
\hline$(0+0<7.59)$ & ACEPTADO & $\begin{array}{c}(0+0 \\
<<7.59\end{array}$ & ACEPTADO \\
\hline
\end{tabular}

Tabla 11 Criterio de decisión embarque 2

Fuente: Elaboración propia 
De acuerdo a los resultados anteriores, podemos ver que la inspección de tela, cumple los requisitos respecto a la variable "ancho" al encontrarse dentro de los LE (45.2" a 46.4”), así como también en relación a la variable "onzaje" cuyos registros de las muestras recolectadas, se encontraron dentro de los LE $(1.8 \mathrm{Oz} / \mathrm{Yd} 2$ a 2.2 $\mathrm{Oz} / \mathrm{Yd} 2)$, por lo que dicho lote se envía al almacén de materia prima.

\section{Embarque 3}

El embarque de tela tubular blanca mantiene un total de 66 rollos y de acuerdo con el muestreo de aceptación, se deberá inspeccionar la cantidad de 5 rollos, de acuerdo al nivel 6 de inspección, con el código $\mathrm{D}$, para finalmente los resultados compararlos con el valor de $\mathrm{M}$ que equivale a 9.80 .

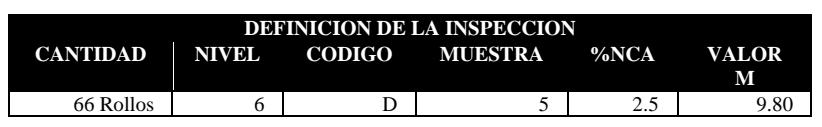

Tabla 12 Parámetros de inspección, embarque 3 Fuente: Elaboración propia

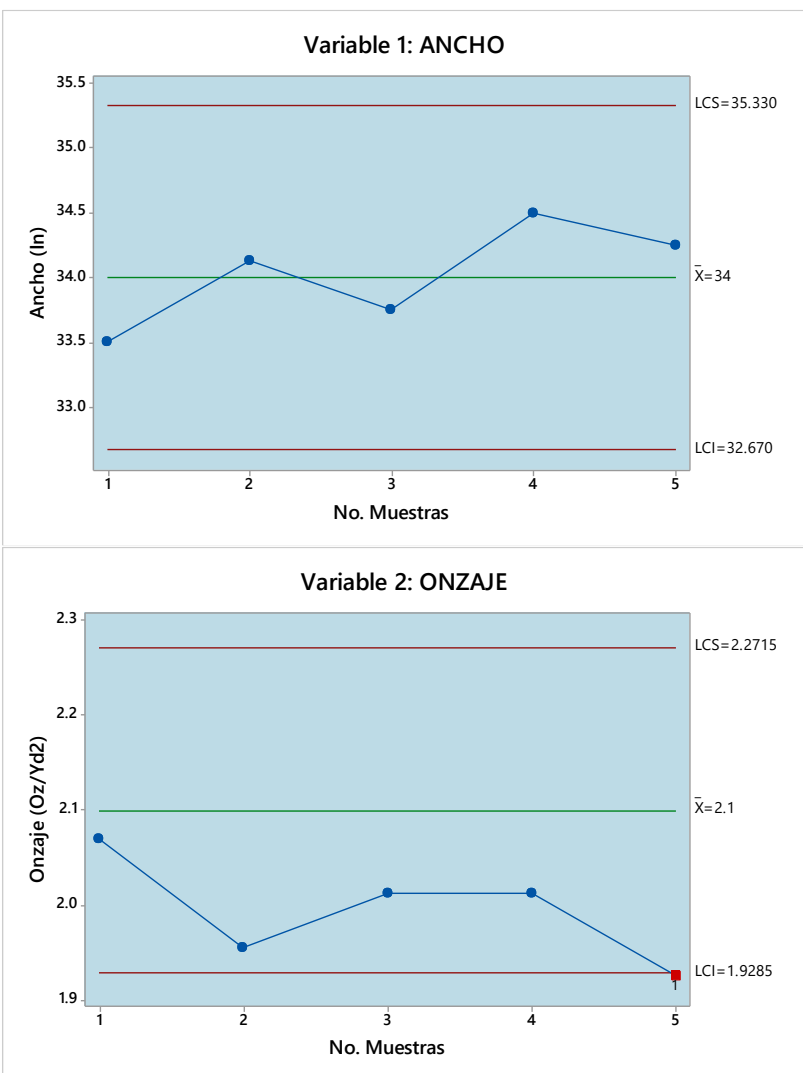

Figura 10 Gráficos de inspección, embarque 3 Fuente: Elaboración propia

\begin{tabular}{|l|r|r|l|r|r|r|}
\hline \multicolumn{7}{|c|}{ ANALISÍS DE LA INSPECCION } \\
\hline Variables & Ancho & Onzaje & & Variables & \multicolumn{1}{c|}{ Ancho } & Onzaje \\
\hline LSE $=$ & 35.330 & 2.2715 & & ZES $=$ & 3.2706 & 4.9375 \\
\hline LIE $=$ & 32.670 & 1.9285 & & ZEI $=$ & 3.3959 & 1.1875 \\
\hline$\dot{\mathrm{X}}=$ & 34.025 & 1.995 & & PS $=$ & 0 & 0 \\
\hline $\mathrm{s}=$ & 0.399 & 0.056 & & $\mathbf{P I}=$ & 0 & 10.76 \\
\hline
\end{tabular}

Tabla 13 Análisis de inspección, embarque 2 Fuente: Elaboración propia

\section{TOMA DE DECISIONES}

Sí (PS + PI < M), entonces el lote se ACEPTA, de lo contrario el lote

se RECHAZA.

\begin{tabular}{|c|c|c|c|}
\hline & & ONZAJE & \\
\hline$<9.80)$ & ACEPTADO & $(0+10.76<$ & RECHAZAD \\
\hline
\end{tabular}

Tabla 14 Criterio de decisión embarque 3

Fuente: Elaboración propia

Como podemos ver en el plan de muestreo anterior, la tela tubular blanca, sólo cumplió con una de las dos variables medidas, en el caso de la variable "ancho" se encontró dentro de los LE (32.6" a 35.3"), sin embargo, la variable "onzaje" se ubicó por debajo de los LE $(1.9 \mathrm{Oz} / \mathrm{Yd} 2$ a $2.2 \mathrm{Oz} / \mathrm{Yd} 2)$, por lo que dicho lote fue rechazado al no cumplir los parámetros anteriores.

En total se evaluaron 27 embarques durante el segundo semestre del año 2019, dichos resultados servirán de base en el proceso de mejora continua, dentro de la siguiente etapa de esta investigación.

\section{Etapa: Actuar}

En la etapa anterior se logró desarrollar la implementación del plan de muestreo, logrando excelentes resultados a favor del correcto desarrollo en la inspección de materia prima, es por ello que se aprobó tal metodología, para establecerse en la planta.

Como siguiente paso se capacita al personal del área de inspección y almacén, en la ejecución del plan de muestreo, derivado de esto se generan dos nuevos procedimientos denominados: "Plan de muestreo de materias primas y material de empaque” y "XXX-CC-O1007 Procedimiento de inspección y recibo de MP y Empaque”.

En relación a la evaluación de proveedores, hubo un incremento el número de estos, sin embargo no todos cumplieron con los acuerdos y especificaciones requeridas. La siguiente gráfica presenta la evaluación realizada en el segundo semestre del año 2019: 
Evaluacion de Proveedores 2019

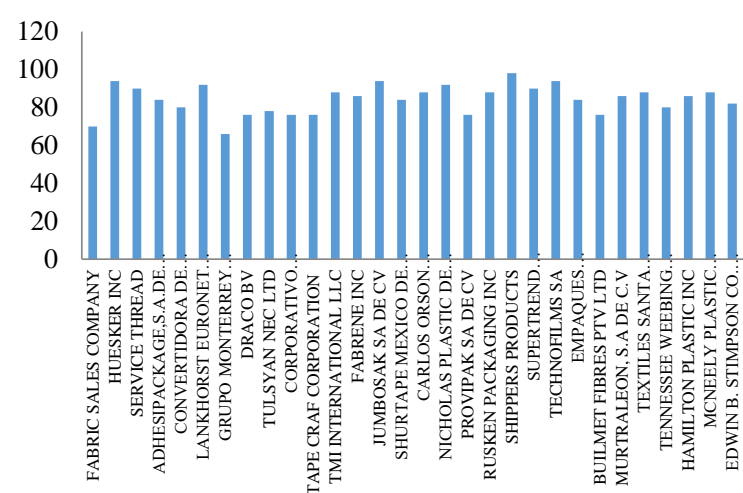

Figura 11 Evaluación de proveedores 2019-2

Fuente: Elaboración propia

Derivado de lo anterior se pudo identificar que el $15.09 \%$ del total de los proveedores obtuvo una calificación de cumplimiento con las especificaciones en sus insumos, por debajo del $78 \%$, por lo que se deberá evaluar si continúan colaborando con la planta, debido a que sus materiales son poco confiables.

\section{Análisis de resultados}

Se logró la implementación del plan de muestreo por variables, lo cual trajo grandes resultados al proceso de inspección de materias primas y materiales. Dentro de esto, el mecanismo de inspección visual ayudó a garantizar que se cumpliera con la calidad e inocuidad de los insumos. En la siguiente figura podemos ver cómo eran recibidos estos, antes de la inspección visual:

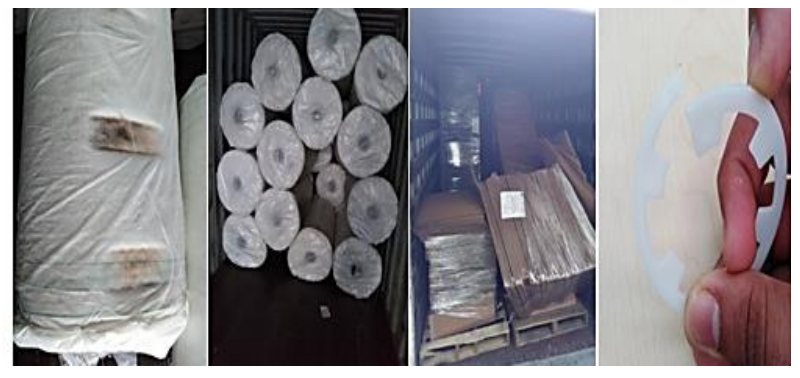

Figura 12 Materia Prima recibida en el Semestre-1 2019 Fuente: SIG

Como podemos ver los materiales eran recibidos sin los mínimos cuidados en su manejo $\mathrm{y}$ traslado, lo cual afectaba directamente su calidad e inocuidad, generando desperdicio para la planta. Con la herramienta de inspección visual, esto mejoro considerablemente, tal como podemos ver:

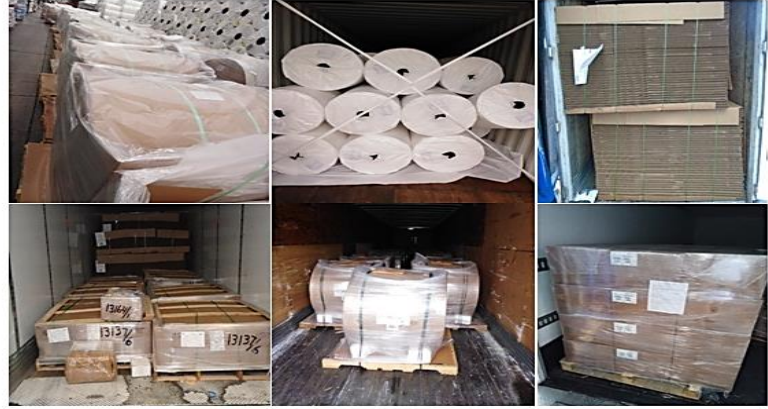

Figura 13 Materia Prima recibida en el Semestre-2 2019 Fuente: SIG.

Esta mejora va de la mano de la evaluación a los proveedores, lo que permite tener con ellos una retroalimentación sobre el nivel de calidad de sus insumos, así mismo ellos reafirman su compromiso de cumplir con los requerimientos solicitados. En la siguiente gráfica podemos ver el comparativo de la evaluación realizada en el año 2018 y 2019:

COMPARATIVO DE EVALUACION DE PROVEEDORES DEL 2018 Y 2019

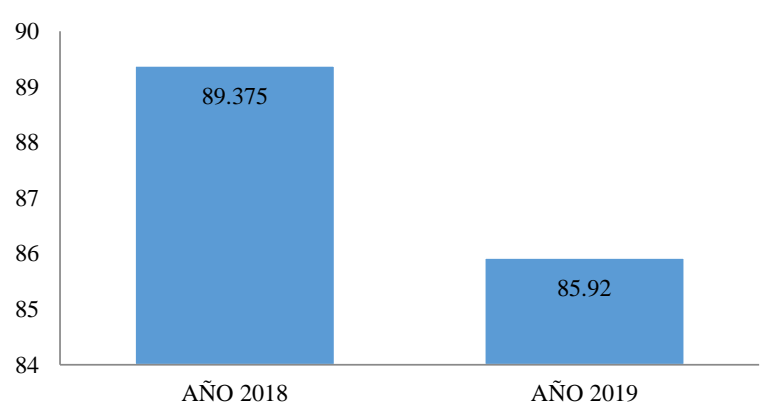

Figura 14 Gráfica comparativa de evaluación de proveedores 2018 vs 2019

Fuente: Elaboración propia

Como podemos apreciar la calificación de desempeño de los proveedores disminuyó $3.455 \%$ debido a que en el año 2018, no se consideraba el instrumento de inspección visual que se implementó recientemente, ya que este último evalúa el grado de cumplimiento respecto a la calidad e inocuidad de los materiales recibidos.

Respecto al plan de muestreo por variables, se concretó su implementación durante la cual se evaluaron 27 lotes, de los cuales sólo 4 presentaron defectos de embarque o daños por traslado. El uso del plan de muestreo contribuyó en gran medida en la mejora de los procesos y productos, así como garantizar la calidad de estos ante los clientes. 
Durante la implementación de esta investigación se logró disminuir el porcentaje de producto defectuoso a un 13\%, y en consecuencia el Nivel de calidad aceptable incrementó pasó del $79 \%$ a un $87 \%$, tal como lo vemos en el siguiente gráfico:

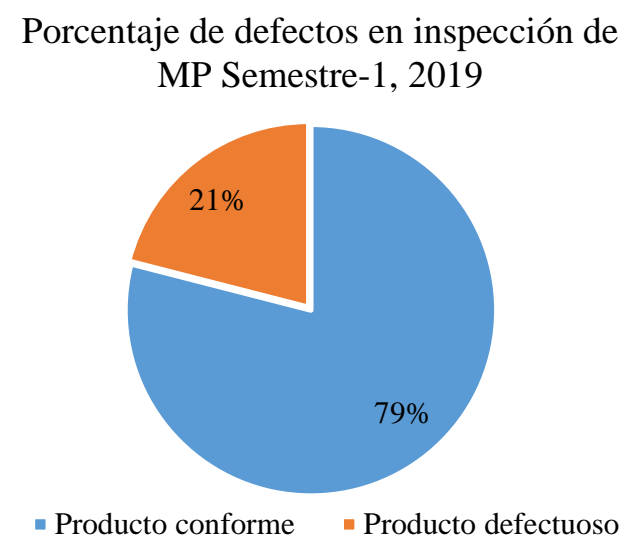

Porcentaje de defectos en inspección de MP Semestre-2, 2019

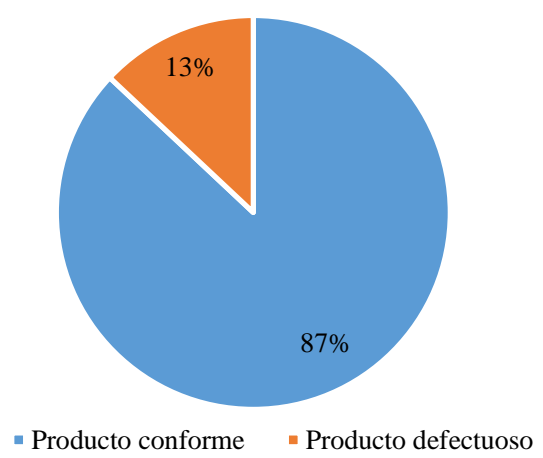

Figura 15 Gráficas comparativas -Porcentaje de defectos en inspección

Fuente: Elaboración propia

\section{Conclusiones}

En este proyecto se ha aplicado la metodología del ciclo de mejora continua (PHVA), para solventar la necesidad de una empresa, la cual era reducir los defectos que se generaban al recibir e inspeccionar la materia prima; dichos defectos hacían que los productos no cumplieran con la calidad requerida, además de que ocasionaban problemas con otros procesos y maquinaria usada en la fabricación de dichos productos.

Las técnicas de muestreo han demostrado ser una valiosa herramienta en el control de calidad, independientemente el proceso o área en que se aplique, por lo que su uso debe ser considerado como estratégico para garantizar la satisfacción de los requerimientos de los clientes, tanto internos como externos.

\section{Referencias}

Barra, R. (2012). Manual de Calidad. Sudamérica.

Feigenbaum, Armand V. (1991). Control Estadístico de la calidad. Editorial Compañía editorial Continental. México.

Gutiérrez Alba, J. (1996). Aspectos Metodológicos de Muestreo de Aceptación con Énfasis en Bulk Sampling. Xalapa, Ver.

Gutiérrez Pulido, H., \& de la Vara Salazar, R. (2013). Control estadístico de la Calidad y Seis Sigma. México: McGraw-Hill.

ISO 3951-1, 2. (2013). Procedimientos para la inspección por la variable -Parte 1: Especificación para los planes de muestreo por límite de calidad de aceptación (AQL) para la inspección lote por lote para características y un grado de desviación máxima para el control del AQL.

ISO 9000, 2. (2015). Sistema de Gestion de la calidad- Fundamentos y Vocabulario.

ISO 9001, 2. (2015). Sistema de Gestion de la Calidad - Mejora Continua.

Kleeberg Hidalgo, Fernando; Ramos Ramírez, Julio Cesar (2009). Aplicación de las técnicas de muestreo en los negocios y la industria. Universidad de Lima.

Luna Valenzuela, Roxana. (2011). Muestreo de aceptación de lotes por atributos en las artesanías de la región de cusco. Universidad Nacional de San Antonio Abad del Cusco. Perú.

Melo Cala, Pavel (2013). Diseño del sistema de control de calidad de los diagnosticadores centis en función de los requerimientos de los clientes. Centro de Isotopos. Cuba.

Montgomery, Douglas C. (1996). Probabilidad y Estadística Aplicada a la Ingeniería. México.: McGraw Hill.

Peralta Cruz, Shair Eduardo; Sáenz Castro, Enrique Antonio; Marín Muñoz, Sergio Bautista. (2015). Mejora de la calidad en el proceso de fabricación de chinelas de hule en Rolter S.A. Universidad Nacional de Managua.

HERNÁNDEZ-BENITO, Fernando, GONZÁLEZ-SÓBAL, Martín, GÓMEZ-MÁRQUEZ, Montserrat y SOLÍS-JIMÉNEZ, Miguel Ángel. Mejoramiento de la calidad en la recepción de materias primas, mediante la implementación de un plan de muestreo por variables. Revista de Ingeniería Industrial. 2020 
Rendón Castaño, H. D. (2013). Control Estadistico de Calidad. Medellín.: Universidad Nacional de Colombia.

Ruiz, A., \& Rojas, F. (2006). Muestreo de Aceptacion. Madrid: Universidad Pontificia.

Salinas Martínez, Ana María. (2004). Métodos de muestreo. Ciencia UANL, Monterrey, México.

Sandoval Vásquez, Juan C. (2010). Implementación de un plan de muestreo para establecer parámetros y criterios de aceptación en la elaboración de esponjas de poliuretano. Universidad de San Carlos de Guatemala 